\title{
ERRATUM
}

Jason M. R. Gill · Sara L. Herd · Vandana Vora

Adrianne E. Hardman

\section{Effects of a brisk walk on lipoprotein lipase activity and plasma triglyceride concentrations in the fasted and postprandial states}

Published online: 1 April 2003

(C) Springer-Verlag 2003

\section{Eur J Appl Physiol (2003) 89:184-190}

The introduction of this article stated that 'low density lipoprotein' are a feature of the atherogenic lipoprotein phenotype. This was a misprint and should have stated that 'small dense low density lipoprotein' are a feature of the atherogenic lipoprotein phenotype.

The online version of the original article can be found at http:// dx.doi.org/10.1007/s00421-002-0788-9

J. M. R. Gill ( $\square)$

Department of Pathological Biochemistry,

Glasgow Royal Infirmary,

4th Floor, Queen Elizabeth Building,

Glasgow, G31 2ER, UK

E-mail: j.gill@clinmed.gla.ac.uk

Tel.: + 44-141-2114595

Fax: +44-141-5532558

S. L. Herd · A. E. Hardman

School of Sport and Exercise Sciences,

Loughborough University,

Loughborough, UK

V. Vora

Sheffield Teaching Hospitals Trust,

Sheffield, UK 